\title{
Evolution of Wireless Standards for Health Monitoring
}

\author{
Abdul Saboor, Adeel Mustafa, Rizwan Ahmad, Muneeb Ahmed Khan, Muhammad Haris, Rashid Hameed \\ School of Electrical Engineering and Computer Science, National University of Sciences \\ and Technology (NUST), Islamabad, Pakistan \\ Email:[asaboor.msit15seecs, amustafa.msit15seecs, rizwan.ahmad, mkhan.msit15seecs, \\ mharis.msit15seecs, rashid.hameed]@ seecs.edu.pk
}

\begin{abstract}
Technology is playing a vital role in the improvement of medical field resulting in high life expectancy. The use of wireless networks is one of the modern and efficient ways to monitor health problems remotely. In this context, various wireless monitoring standards are developed to facilities the patient monitoring. Some key standards include IEEE 802.15.4 Low-Rate Wireless Personal Area Network (LR-WPAN), IEEE 802.15.6 Wireless Body Area Network (WBAN) and ETSI smartBAN. These standards consist of multiple sensors that are used to monitor, process and transmit the vitals to the proper destination. Each standard offers some advantages and limitations over the other standard depending on the scenarios. In this paper, all the above-mentioned standards are compared and analyzed on different parameters such as network type, density, functionality, size and energy efficiency.
\end{abstract}

Index Terms-Wireless Health Monitoring, Wireless Body Area Network (WBAN), ETSI smartBAN

\section{INTRODUCTION}

Technology advancements in monitoring solutions have led to increased life expectancy. It is difficult for the patients to avail real-time health monitoring, considering the costs involved and time required to stay in hospitals. The wireless monitoring system is a definite solution to address all the above-mentioned problems [1]. Wireless systems have also motivated and incorporated the other domains such as sports, simulation, E gaming and military applications [2], [3].

IEEE has introduced 802.15.4 Low-Rate Wireless Personal Area Networks (LR-WPAN) in 2003 for industrial applications [4]. These applications include monitoring, controlling and management of houses, offices, industries and agriculture. Due to the large demands of wireless patient monitoring in the past, IEEE 802.15.4 WPAN had been also used as a monitoring standard. It consists of small wireless sensors equipped with a radio transceiver and multiple transducers that are used to collect environmental (or health-related) information. However, IEEE 802.15.4 standard lacks various key factors that are essential for wireless patient monitoring such as life time of sensors [5]. Other parameters are reliability, mobility, low power, sensor size, data rate, latency, interference and lack of traffic flows. Thus, IEEE introduced 802.15.6 Wireless Body Area Network (WBAN) standard to facilitate the growing demands of wireless patient monitoring in 2012 [6]. IEEE 802.15.6 WBAN standard typically supports medical applications but it also covers military, entertainment and sports applications [3], [7], [8]. In order to support a variety of applications, this standard supports the use of three different physical layers. It includes Narrow Band (NB), Ultra Wide Band (UWB) and Human Body Communication (HBC) band. IEEE 802.15.6 WBAN standard is also composed of tiny, robust, ultra-low power, highly reliable and secure on-body, in-body and implanted sensors. It has the provision to support different priority levels of traffic. IEEE 802.15.6 WBAN shares only one Medium Access Control (MAC) to support different flows of different priority sensors; continuous flow, periodic flow and burst mode. Having said that, this standard is not light itself [9] and deficits some important parameters such as very high energy efficiency, Co-existence mechanism in the presence of neighboring Body Area Networks(BANs), latency, packet error correction etc. In addition to that, the implementation of the system is really tough in the presence of numerous functionalities, hence, making the system very complex to implement and evaluate [10]. In this context, the European Telecommunication Standards Institute (ETSI) formed a Technical Committee (TC) name TC smartBAN in 2013. The main agenda of TC was to form a lightweight and low complexity standard. Some technical requirements of the smart BAN include very high energy efficiency, timely access mechanism, optimum Quality of Service (QoS) control and coexistence between other BAN systems. Description of MAC and Physical (PHY) layer defined by ETSI TC is given in [11], [12]. Its target applications include safety monitoring, fall monitoring, sleep monitoring, abnormal Cardiac monitoring, sports monitoring and can also be used in industrial monitoring applications (that required periodic monitoring) [13].

In this paper most commonly used wireless monitoring standards (IEEE 802.15.4 WPAN, IEEE 802.15.6 WBAN, ETSI smart BAN) are analyzed. Key motivation and functionalities of MAC and PHY layers of all the standards are presented. All the standards are further compared to various parameters such as system architecture, network complexity, coexistence management, smart relay support etc. In the end, the most optimal standard for future research is highlighted. To the best of our knowledge, this is the first comparison of health monitoring standards involving smartBAN. Section II of this paper represents the standards. Section III gives a detailed 


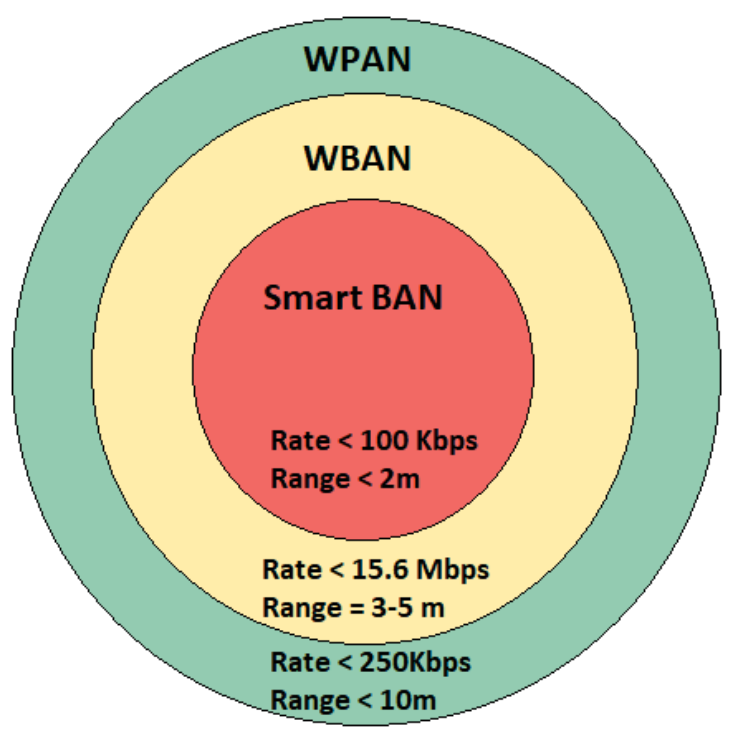

Fig. 1: Approximate transmission Range of Wireless Health Monitoring Standards

comparison and analysis of all the standards and Section IV concludes the work.

\section{WIRELESS HEALTH MONITORING STANDARDS}

IEEE 802.15.4 WPAN, IEEE 802.15.6 and ETSI smart BAN are discussed in this Section. IEEE 802.15.4 supports the range of up to 10 meters while other standards operate in the vicinity of the human body as shown in Fig. 1. The overview of all the standards is given as follows:

\section{A. IEEE 802.15.4 WPAN:}

In the IEEE 802.15.4 WPAN standard, there are two types of network [14]. The first type is a Full Function Device (FFD) that has the capability to communicate with other nodes. An FFD can act as a coordinator (communicate with the whole network) as well as the node. The second type is a very low power Reduced Function Devices (RFD) nodes that can only communicate with the coordinator. IEEE 802.15.4 WPAN operates in beacon-enabled superframe mode for the communications at the MAC layer. Beacons are transmitted after regular intervals that are used to synchronize the nodes with the coordinator and to define the length of the superframe. Overall superframe structure of IEEE 802.15.4 WPAN is shown in Fig. 2 [15]. The superframe is further divided into active and inactive modes. Nodes can go to sleep during the inactive interval to conserve the energy. The mac Superframe Order (SO) and the mac Beacon Order (BO) are the two attributes that are used to calculate various parameters such as the duration/ length of the beacon interval (BI) and the active portion of the superframe respectively. The active portion of superframe consist of 16 slots of equal length used to facilitate the transmissions. Each slot size is equal to $2 \mathrm{So}$

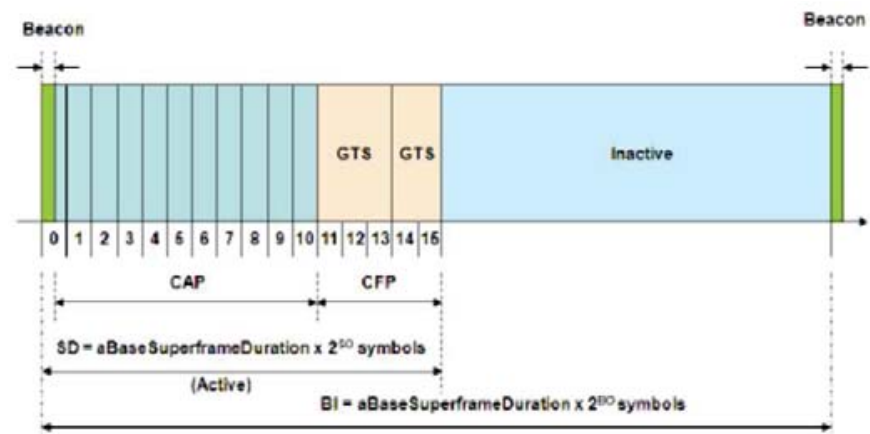

Fig. 2: IEEE 802.15.4 WPAN superframe structure [15]

* aBaseSlotDuration (value of aBaseSlotDuration is equal to 60 symbols). The active portion of the superframe in IEEE 802.15.4 WPAN is divided into three portions: Beacon, Contention Free Period (CFP) and Contention Access Period (CAP). Explanation of superframe will be discussed in section III.

\section{B. IEEE 802.15.6 WBAN:}

The IEEE 802.15.6 WBAN is the combination of tiny, ultralow power, reliable and robust sensors that are placed on, in or inside any subject [8], [16], [17]. In the majority of the cases the human body is the subject, however, it also supports other electronic subjects. There are three types of node defined in IEEE 802.15.6 WBAN based on the functionality. The first type is the general sensors that are used to collect and measure specific parameters from the subjects. These sensors include EEG, EMG, ECG, glucose, motion and temperature sensors. The second type is acuter that is used for communication with the user upon receiving the data. For example, in ubiquitous healthcare applications, of pumping of the correct dose of medicine to the human body is done with the help of acuter [18]. The third type is Personal Device that is used to collect information from sensors and acuter. There is also a second classification of nodes in IEEE 802.15.6 WBAN such as body surface nodes, implanted nodes, relays, coordinator and end nodes. There are three different superframe modes at MAC in IEEE 802.15.6 WBAN as shown in Fig. 3. Basic tasks and comparison of these modes will be discussed in chapter III

\section{ETSI smart BAN}

A smartBAN (ETSI smartBAN) is a low power and simple network that facilitates the wireless connectivity among the nodes and a hub. A hub acts as smartBAN coordinator. BAN cluster is the combination of multiple nodes including sensors, hubs, relays and actuators. There is a high probability of these nodes coming from different manufacturers with different processing power and functionality. This results in heterogeneity in terms of model, data gathering and communication protocols. In ETSI smartBAN, a common semantic approach is introduced to overcome heterogeneity problems. An open data model is used in this approach that is dedicated to smartBAN with the capability of similarity detection and 
TABLE I: General Comparison of Wireless Health Monitoring

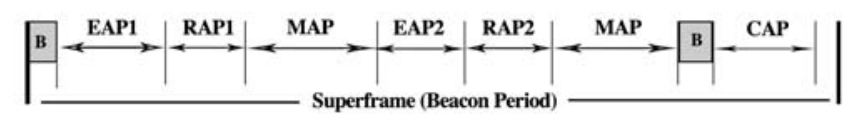
Standards

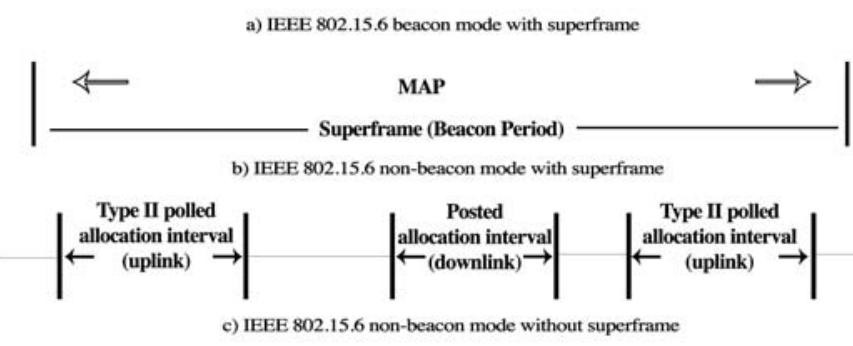

Fig. 3: IEEE 802.15.6 WBAN Superframe Structure [3]

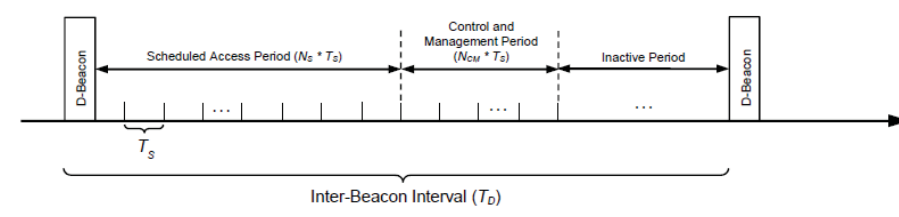

Fig. 4: ETSI smartBAN Superframe Structure [7]

\begin{tabular}{|c|c|c|c|}
\hline Parameter & 802.15 .4 & 802.15 .6 & smartBAN \\
\hline $\begin{array}{l}\text { Network } \\
\text { Topology }\end{array}$ & $\begin{array}{l}\text { Star, Peer-to- } \\
\text { Peer }\end{array}$ & $\begin{array}{l}\text { One or Two } \\
\text { Hop Star }\end{array}$ & Star \\
\hline Coverage & $10 \mathrm{~m}$ & $2-5 m$ & $>2 \mathrm{~m}$ \\
\hline $\begin{array}{l}\text { Smart Relay } \\
\text { Support }\end{array}$ & No & No & Yes \\
\hline $\begin{array}{l}\text { Network Size } \\
\text { (at max) }\end{array}$ & 65000 Nodes & 256 Nodes & 16 Nodes \\
\hline Applications & $\begin{array}{l}\text { Industrial, } \\
\text { Home, } \\
\text { Automation }\end{array}$ & $\begin{array}{l}\text { Medical, } \\
\text { Sports, } \\
\text { Entertainment }\end{array}$ & $\begin{array}{l}\text { Medical, } \\
\text { Sports, } \\
\text { Entertainment }\end{array}$ \\
\hline $\begin{array}{l}\text { Heterogeneity } \\
\text { Management }\end{array}$ & No & No & Yes \\
\hline $\begin{array}{l}\text { Coexistence } \\
\text { Management } \\
\text { by } \\
\text { Coordinator }\end{array}$ & Low & Low & High \\
\hline Node Size & $\begin{array}{l}\text { Relatively } \\
\text { Big }\end{array}$ & Small & Small \\
\hline Node Type & In/on-body & $\begin{array}{l}\text { In/on-body, } \\
\text { Implanted }\end{array}$ & $\begin{array}{l}\text { In/on-body, } \\
\text { Implanted }\end{array}$ \\
\hline $\begin{array}{l}\text { Node } \\
\text { Replacement }\end{array}$ & Easy & Hard & Hard \\
\hline $\begin{array}{l}\begin{array}{l}\text { Energy } \\
\text { ciency }\end{array} \\
\end{array}$ & $\begin{array}{l}\text { Comparatively } \\
\text { low }\end{array}$ & Average & High \\
\hline
\end{tabular}

conflict resolution [19]. In addition, ETSI smartBAN provides enhanced reliability and productivity, minimized listening period of nodes and the asymmetrical relation between nodes and the hub where the hub performs most functionality [11] ETSI smartBAN follows a unique two channel concept control and data traffic as shown in Fig. 4.

The overall comparison of all the standards is presented in Section III.

\section{COMPARISON AND ANALYSiS OF HEALTH MONITORING STANDARDS}

The aim of all the standards mentioned in Section II is to provide reliable health monitoring to the patients, facilitates the patients that cannot afford to stay longer in the hospital and behave in a proactive manner to reduce the severity of chronic diseases. These factors result in an improved QoS and Quality of Life (QoL). However, each standard has some limitation associated with it. Therefore, in this section, a detailed comparison of IEEE 802.15.4 WPAN, IEEE 802.15.6 WBAN and ETSI smartBAN is presented.

\section{A. General Comparison:}

In general, all these standards differ with each other on the following parameters:

- Network Topology: There is a support of peer-to-peer and star topology in IEEE 802.15.4 standard. In a star topology, nodes can only communicate with the coordinator while any node can communicate with each other or the hub in a peer-to-peer topology. IEEE 802.15.6 WBAN supports one or two hop star topology while ETSI smartBAN standard only supports start topology.

- Coverage: WPAN has the maximum coverage area as it was developed for industrial applications. It covers the range of $10 \mathrm{~m}$. The aim of WBAN and smartBAN is to operate against the vicinity of the human body so both the standards cover $2-5 \mathrm{~m}$ and less than $2 \mathrm{~m}$ range respectively.

- Smart Relay: ETSI smartBAN has the provision of smart relay that makes this standard robust and offers better performance. Other standards dont support the smart Relay.

- Network Size: IEEE 802.15.4 WPAN standard supports huge (up to 65000) number of sensors. IEEE 802.15.6 WBAN can support a maximum of 256 nodes. ETSI smartBAN is the simplest standard with the support of up to 16 nodes (typically 8 nodes) [13].

- Applications: WPAN generally supports industrial, home and automation related application. Other standards usually target medical applications such as patient monitoring, telehealth monitoring and safety monitoring. In addition, both standards also support fitness, entertainment and sports related applications.

- Heterogeneity Management: ETSI smartBAN is the only standard that supports heterogeneity management in the presence of different types of sensors. This is one of the major reason to shift from WBAN standard to smartBAN standard.

- Coexistence management by coordinator: ETSI smartBAN has the high support of coexistence management as compared to other standards.

- Node Size: Nodes is in WBAN and smartBAN are very lightweight and small. However, in WPAN, small size is preferred but it is not essential.

- Node Type: All the standard has the provision to support 
TABLE II: MAC Comparison of Wireless Health Monitoring Standards

\begin{tabular}{|l|l|l|l|}
\hline Parameter & $\mathbf{8 0 2 . 1 5 . 4}$ & $\mathbf{8 0 2 . 1 5 . 6}$ & smartBAN \\
\hline $\begin{array}{l}\text { Superframe } \\
\text { Phases }\end{array}$ & $\begin{array}{l}\text { CAP, CFP, In- } \\
\text { active }\end{array}$ & $\begin{array}{l}\text { EAP, RAP, } \\
\text { MAP, CAP }\end{array}$ & $\begin{array}{l}\text { SAP, CMP, } \\
\text { Inactive }\end{array}$ \\
\hline $\begin{array}{l}\text { Access Mech- } \\
\text { anisms }\end{array}$ & $\begin{array}{l}\text { TDMA, } \\
\text { Slotted } \\
\text { CSMA/CA }\end{array}$ & $\begin{array}{l}\text { Polling, } \\
\text { Slotted } \\
\text { Aloha, } \\
\text { CSMA/CA }\end{array}$ & $\begin{array}{l}\text { TDMA, } \\
\text { Slotted } \\
\text { Aloha, Multi- } \\
\text { use }\end{array}$ \\
\hline $\begin{array}{l}\text { Provision for } \\
\text { Emergency } \\
\text { Traffic }\end{array}$ & No & Yes & Yes \\
\hline $\begin{array}{l}\text { Beacon } \\
\text { Transmission }\end{array}$ & Yes & Yes & $\begin{array}{l}\text { Yes (Except } \\
\text { Mode3) }\end{array}$ \\
\hline $\begin{array}{l}\text { User } \\
\text { Priorities }\end{array}$ & 1 & 8 & 4 \\
\hline $\begin{array}{l}\text { Data Rates } \\
\text { Up to 250 }\end{array}$ & $\begin{array}{l}\text { Up to 15.6 } \\
\text { Mbps }\end{array}$ & $>100$ Kbps \\
\hline
\end{tabular}

on/in-body sensors. IEEE 802.15.6 WBAN also ETSI smartBANs also support implanted devices.

- Node replacement: Implanted nodes replacement is really challenging task in WBAN and smartBANs. Replacement of body nodes can be done easily in all the standards.

The overall comparison is shown in table I.

\section{B. Comparison at MAC layer:}

MAC layer of all the above-mentioned standards is different in the following parameters:

- General Structure and Access Mechanisms: The superframe of IEEE 802.15.4 is divided into three portions: a beacon, active portion and inactive portion. Active portion is subdivided into CAP and CFP. Slotted Carrier Sense Multiple Access with Collision Avoidance (CSMA/CA) is used in CAP and Time Division Multiple Access (TDMA) is used CFP. Guaranteed Time Slots (GTSs) are used in CFP to facilitate the nodes with a low latency and specific data rate requirements. IEEE 802.15.6 supports three different types of superframes. The first mode is beacon mode with superframe boundaries consisting of a beacon, two Exclusive Access Phases (EAPs), two Random Access Phases (RAPs), two Managed Access Phases (MAPs) and one CAP [20]. EAP is used to facilitates the data of the highest priority. CSMA/CA or slotted Aloha is used in both EAPs, RAPs and CAP. Polling is used in the MAP for uplink, downlink or bi-link resource allocation. The second mode is Beacon Mode without superframe boundaries that is entirely consisting of the MAP. In the third mode (Non-Beacon Mode without superframe boundaries) only type II polled allocation is provided by the coordinator. In ETSI smartBAN, the length of the superframe is marked by transmitting two Control-Beacons (C-Beacons). The boundaries of CBeacons is marked by Data- Beacons (D-Beacons) for the transmission of data. The time between D-Beacons is distributed in a Scheduled Access Period (SAP), a Control
TABLE III: PHY Comparison of Wireless Health Monitoring Standards

\begin{tabular}{|l|l|l|l|}
\hline Parameter & $\mathbf{8 0 2 . 1 5 . 4}$ & $\mathbf{8 0 2 . 1 5 . 6}$ & smartBAN \\
\hline $\begin{array}{l}\text { Operating } \\
\text { Frequency }\end{array}$ & $\begin{array}{l}2.400- \\
2.4835 \\
\mathrm{GHz}\end{array}$ & $\begin{array}{l}2.360-2.400 \\
\mathrm{GHz}, 2.400-\end{array}$ & $\begin{array}{l}2.401-2.481 \\
\mathrm{GHz}\end{array}$ \\
\hline $\begin{array}{l}\text { Number of } \\
\text { Channels }\end{array}$ & 16 & 39,79 & 40 \\
\hline $\begin{array}{l}\text { Channel } \\
\text { Bandwidth }\end{array}$ & $2 \mathrm{MHz}$ & $1 \mathrm{MHz}$ & $2 \mathrm{MHz}$ \\
\hline $\begin{array}{l}\text { Symbol Rate } \\
\text { Modulation }\end{array}$ & 0.0625 & $0.6 \mathrm{MSps}$ & $1 \mathrm{MSps}$ \\
MScheme & OQPSK & $\begin{array}{l}\text { DBPSK, } \\
\text { DQPSK }\end{array}$ & GFSK \\
\hline Preamble Size & $32 \mathrm{bits}$ & $90 \mathrm{bits}$ & $16 \mathrm{bits}$ \\
\hline Header Size & $8 \mathrm{bits}$ & $31 \mathrm{bits}$ & $40 \mathrm{bits}$ \\
\hline
\end{tabular}

and Management Period (CMP) and an Inactive Period (IP). Scheduled channel access and slotted channel access is used in SAP and CMP respectively. Multiuse channel access can be used in both SAP and CMP.

- Provision for Emergency Traffic: Both IEEE 802.15.6 WBAN and ETSI smartBAN has the provision for emergency or high priority traffic.

- Beacon Transmission: For synchronization, a beacon is used at the start of the superframe in all the standards except superframe mode 3 in IEEE 802.15.6 WBAN.

- User Priorities: IEEE 802.15.6 defines eight different data priorities while only 4 priorities are present in smartBAN. IEEE 802.15.4 standard can support only a single priority.

- Security Level: IEEE 802.15.4 WPAN generally provides low-level security (Application Dependent). IEEE 802.15.6 WBAN provides three level levels of security including encryption and authentication.

- Data Rates: IEEE 802.15.6 WBAN can support the highest data rates up to $15.6 \mathrm{Mbps}$. ETSI smartBAN can support data rate less than 100 Kbps. IEEE 802.15.4 WPAN can support the data rate up to $250 \mathrm{Kbps}$.

Table II gives a snapshot of overall comparison at MAC layer.

\section{Comparison at PHY layer:}

PHY layer parameters of IEEE 802.15.4 WPAN (2.4 GHz), IEEE 802.15.6 WBAN (2.4 GHz) and ETSI smartBAN are shown in Table III and compared as follows:

- Operating Frequency: The operating frequency of IEEE 802.15.4 WPAN (2.4 GHz) is in the range of $2.400 \mathrm{GHz}$ to $2.4835 \mathrm{GHz}$. IEEE 802.15.6 WBAN operates in 2.360$2.400 \mathrm{GHz}$ and $2.400-2.4835 \mathrm{GHz}$ frequency range. ETSI smartBAN operates between $2.401 \mathrm{GHz}$ to $2.481 \mathrm{GHz}$.

- Number of Channels: There is a maximum of 16 and 40 channels in 802.15.4 and smartBAN standard respectively. IEEE 802.15.6 standard has 39 channels (in $2.3602 .400 \mathrm{GHz}$ ) and 79 channels (in 2.400-2.4835 $\mathrm{GHz})$. 
- Channel Bandwidth: The bandwidth of each channel in IEEE 802.15.4 and ETSI smartBAN is $2 \mathrm{MHz}$. While the channel bandwidth in IEEE 802.15.6 WBAN is $1 \mathrm{MHz}$.

- Symbol Rate: IEEE 802.15.4 WPAN has the symbol rate of 0.0625 MSps. IEEE 802.15.6 has the symbol rate of $0.6 \mathrm{MSps}$ and ETSI smartBAN has the rate of $1 \mathrm{MSps}$ [21].

- Modulation Scheme: Offset Quadrature Phase-Shift Keying (OQPSK) is used in WPANs. WBANs use Differential Binary Phase-Shift Keying (DBPSK) and Differential Quadrature Phase-Shift Keying (DQPSK). Gaussian Frequency Shift Keying (GFSK) modulation scheme is used in ETSI smartBAN.

- Preamble and Header Size: PHY Protocol Data Unit (PPDU) of 802.15.4 has a preamble of 32 bits and header of 8 bits. PPDU of 802.15 .6 consists of 90 bit long preamble and 31 bit long header. Preamble size in smartBAN is 16 bits and the header size is 40 bits.

From the analysis, it is observed that IEEE 802.15.4 WPAN is not efficient enough for developing healthcare applications. This is mainly due to the low energy efficiency, node size and provision for the only type of traffic. IEEE 802.15.6 WBAN standard owns some essential features required for an efficient health monitoring system. This standard supports the highest data rates, accommodates the emergency traffic in a good manner, supports multiple frequency bands and multi-hop network topology. However, 802.15.6 lacks some key parameters such as heterogeneity management and less efficient coexistence management. Furthermore, this standard is really complex for physical implementation and offers no commercial application solely based on the standard until this date. In reference to all the above analysis, ETSI smartBAN is the optimal choice for the researchers, designers and developers working on wireless health monitoring applications due to its light and less complex nature.

\section{Conclusion:}

Wireless health monitoring systems are devising new trends in the medical field. In this context, a study on various standards that support wireless monitoring is presented. From the analysis, IEEE 802.15.6 WBAN and ETSI smart BAN are found to be optimal for providing medical health care services as compared to IEEE 802.15.4 WPAN due to node size, energy consumption and etc. However, the flexibility offered by the IEEE 802.15.6 standard makes its implementation challenging. Therefore, ETSI smartBAN is the best standard for the development of new health-related services. Our future research aims to investigate the research challenges in ETSI smartBAN.

\section{REFERENCES}

[1] S. Junnila, H. Kailanto, J. Merilahti, A.-M. Vainio, A. Vehkaoja, M. Zakrzewski, and J. Hyttinen, "Wireless, multipurpose in-home health monitoring platform: Two case trials," IEEE transactions on information technology in biomedicine : a publication of the IEEE Engineering in Medicine and Biology Society, vol. 14, pp. 447-55, 122009.
[2] M. Patel and J. Wang, "Applications, challenges, and prospective in emerging body area networking technologies," IEEE Wireless Communications, vol. 17, no. 1, pp. 80-88, February 2010.

[3] A. Saboor, R. Ahmad, W. Ahmed, A. K. Kiani, Y. L. Moullec, and M. M. Alam, "On research challenges in hybrid medium access control protocols for ieee 802.15.6 wbans," IEEE Sensors Journal, 2018.

[4] Wireless medium access control (MAC) and physical layer (PHY) specifications for low rate wireless personal area networks (WPANs) (amendment of IEEE Std 802.15.4-2003). IEEE standard 802.15.4, 2006.

[5] M. Ghamari, B. Janko, R. S. Sherratt, W. Harwin, R. Piechockic, and C. Soltanpur, "A survey on wireless body area networks for ehealthcare systems in residential environments," Sensors, vol. 16, no. 6, 2016.

[6] IEEE Standard for Local and Metropolitan Area Networks-Part 15.6: Wireless Body Area Networks. IEEE Std 802.15.6, 2012.

[7] M. Salayma, A. Al-Dubai, I. Romdhani, and Y. Nasser, "Wireless body area network (wban): A survey on reliability, fault tolerance, and technologies coexistence," ACM Comput. Surv., vol. 50, no. 1, Mar. 2017.

[8] S. Movassaghi, M. Abolhasan, J. Lipman, D. Smith, and A. Jamalipour, "Wireless body area networks: A survey," IEEE Communications Surveys Tutorials, vol. 16, no. 3, pp. 1658-1686, Third 2014.

[9] M. Hmlinen, T. Paso, L. Mucchi, M. Girod-Genet, J. Farserotu, H. Tanaka, W. H. Chin, and L. N. Ismail, "Etsi tc smartban: Overview of the wireless body area network standard," in 2015 9th International Symposium on Medical Information and Communication Technology (ISMICT), March 2015, pp. 1-5.

[10] R. Matsuo, T. Nabetani, H. Tanakay, W. H. Chin, and S. Subramani, "Performance of simple and smart phy/mac mechanisms for body area networks," in 2015 IEEE International Conference on Communications (ICC), June 2015, pp. 501-506.

[11] Smart Body Area Network (SmartBAN); Low Complexity Medium Access Control (MAC) for SmartBAN. ETSI TS 103325 (V1.1.1) (04-2015).

[12] Smart Body Area Network (SmartBan); Enhanced Ultra-Low Power Physical Layer. ETSI TS 103325 (V1.1.1) (04-2015).

[13] Smart Body Area Networks (SmartBAN); System Descriptionr. ETSI TR 103394 V1.1.1 (2018-01).

[14] Y. Xiao, H.-H. Chen, B. Sun, R. Wang, and S. Sethi, "Mac security and security overhead analysis in the ieee 802.15.4 wireless sensor networks," EURASIP J. Wirel. Commun. Netw., vol. 2006, no. 2, Apr. 2006.

[15] S. Mohanty and S. Patra, "Performance analysis of quality of service parameters for ieee 802.15.4 star topology using manet routing," in Proceedings of the International Conference and Workshop on Emerging Trends in Technology, ser. ICWET'10, 2010, pp. 115-120.

[16] K. Takabayashi, H. Tanaka, C. Sugimoto, K. Sakakibara, and R. Kohno, "Performance evaluation of a quality of service control scheme in multihop wban based on ieee 802.15.6," Sensors, vol. 18, no. 11, p. 3969 2018.

[17] M. M. Alam and E. B. Hamida, "Surveying wearable human assistive technology for life and safety critical applications: Standards, challenges and opportunities," Sensors, vol. 14, no. 5, pp. 9153-9209, 2014.

[18] S. Wang and J. Park, "Modeling and analysis of multi-type failures in wireless body area networks with semi-markov model," IEEE Communications Letters, vol. 14, no. 1, pp. 6-8, January 2010.

[19] M. Girod-Genet, L. Nachabe Ismail, and B. El Hassan, "ETSI TS 103 378: Smart Body Area Network (SmartBAN) : unified data representation formats, semantic and open data model," Research Report, Dec. 2015.

[20] A. Saboor, R. Ahmad, W. Ahmed, and M. Alam, "A unique backoff algorithm in ieee 802.15.6 wban," in 2018 IEEE 88th Vehicular Technology Conference (VTC Fall), 2018.

[21] H. Viittala, L. Mucchi, M. Hmlinen, and T. Paso, "Etsi smartban system performance and coexistence verification for healthcare," IEEE Access, vol. 5, pp. 8175-8182, 2017. 